\title{
Contaminación orgánica por coliformes, Nitrógeno y Fósforo en los ecosistemas acuáticos de la cuenca Ayuquila-Armería, Jalisco, México
}

\author{
Organic contamination by coliforms, Nitrogen and Phosphorus in the acuatic ecosistems of the \\ Ayuquila-Armeria basin, Jalisco, Mexico
}

\begin{abstract}
Oscar Raúl Mancilla-Villa1*, Larissa Gómez-Villaseñor², José Luis Olguín-Lopez ${ }^{2}$, Rubén Darío Guevara-Gutiérrez², Omar Hernández-Vargas', Héctor Manuel Ortega-Escobar ${ }^{3}$, Héctor Flores-Magdaleno ${ }^{3}$, Álvaro Can-Chulim ${ }^{4}$, Edgar Iván Sánchez-Bernal ${ }^{5}$, Elia Cruz-Crespo ${ }^{4}$, Carlos Palomera-García ${ }^{2}$

Universidad de Guadalajara, Centro Universitario de la Costa Sur, Departamento de Producción Agrícola. Av. Independencia Nacional \#151 C.P. 48900, Autlán, Jalisco, México

2 Departamento de Ecología y Recursos Naturales, Ingeniería en Recursos Naturales y Agropecuarios. Universidad de Guadalajara, Centro Universitario de la Costa Sur, Av. Independencia Nacional \#151 C.P. 48900, Autlán, Jalisco, México

Hidrociencias, Colegio de Postgraduados, Montecillo, México.

${ }^{4}$ Universidad Autónoma de Nayarit, Tepic, Nayarit.

5 Universidad del Mar, Oaxaca Ciudad Universitaria, Puerto Ángel, Distrito de San Pedro, C.P.70902. Pochutla, Oaxaca, México.
\end{abstract}

\section{RESUMEN}

La cuenca del río Ayuquila-Armería se encuentra dentro de la clasificación de cuencas prioritarias de México, dada su alta diversidad, alto grado de alteración de la dinámica funcional y los niveles de presión social que presenta, por ende, es necesario realizar un análisis de la calidad del agua dentro de la cuenca. El objetivo fue evaluar la contaminación orgánica, realizando dos periodos de muestreo en temporada de lluvia (2015), y seca (2016), en 18 sitios de la cuenca. Se implementó el análisis integral de variables físicoquímicas y biológicas. El $90 \%$ de los sitios presento eutrofización dadas las concentraciones de $\mathrm{P}_{\text {Total }}$ el 77 \% superó el límite permisible para las concentraciones de $\mathrm{N}_{\text {Total }}$ i el IIB evidencio que el $19 \%$ de los sitios presenta un alto grado de contaminación orgánica; en la temporada lluviosa se encontraron las mayores concentraciones de UFC (18 a $190 \mathrm{UFC} / \mathrm{mL}$ ) y un $50 \%$ de los sitios superó el límite permisible (10 UFC/mL). La influencia de los afluentes tributarios al cauce principal aumenta drásticamente en la temporada de lluvias, se incrementa los caudales y empobrece la calidad del agua superficial por el arrastre de contaminantes desde las zonas rurales y urbanas. Palabras clave: nutrientes, contaminación, macroinvertebrados, coliformes, Índice de Integridad Biótica.

\section{ABSTRACT}

The Ayuquila-Armería river basin is within the priority basins classification in Mexico, given its high diversity, and due to its high degree of functional dynamics alteration and levels of social pressure it presents; therefore, it is necessary to carry out an analysis of water quality within the basin. The objective was to evaluate organic contamination, carrying sampling campaigns in two seasons, one in the rainy (2015) and the other in the dry (2016) season, at 18 sites in the basin, through the implementation of a comprehensive analysis of physicochemical and biological variables. According to $\mathrm{P}_{\text {Total }}$ concentrations, $90 \%$ of the sites presented eutrophication, exceeding $77 \%$ of them the permissible limit for $\mathrm{N}_{\text {Total }}$ concentrations. The IIB showed that $19 \%$ of the sites present a high degree of organic contamination, with highest CFU concentrations ( 18 to $190 \mathrm{CFU} / \mathrm{mL}$ ) found in the rainy season and $50 \%$ of the sites exceeding the permissible limit (10 CFU $/ \mathrm{mL}$ ). The influence of tributary influents to the main channel increases drastically in the rainy season, increasing flows and impoverishes the surface water quality by pollutants carryover from rural and urban areas.

Keywords: nutrients, pollution, macroinvertebrates, coliforms, biotic integrity index.

\section{INTRODUCCIÓN}

El recurso hídrico (agua) es uno de los elementos esenciales para la vida, y en los ecosistemas del planeta, ya que interviene en muchos de los procesos ambientales y está relacionado con su buen funcionamiento, esto propicia la generación de servicios ambientales de los que en gran medida depende la salud humana y el desarrollo económico de las naciones (Sierra, 2011). Más de dos terceras partes de la superficie de la tierra están cubiertas de agua, de la cual, $2.5 \%$ es agua dulce en forma sólida en su mayoría (glaciares) o se encuentra en depósitos subterráneos, por lo que sólo $0.4 \%$ de la misma está disponible para actividades humanas (Castro et al., 2014), no obstante, esta mínima porción de agua dulce disponible, sufre cada vez más cambios negativos en las últimas décadas debido a las presiones humanas (Castillo y Vera, 2016).

Para este estudio el enfoque de cuenca es importante, ya que las cuencas hidrográficas son divisiones naturales del paisaje y para muchos propósitos componen el contexto idóneo para la planeación y gestión de los recursos naturales; su estructura y funcionamiento sustentan un equilibrio frágil y dinámico, producto de la interacción entre sus componentes,
*Autor para correspondencia: Oscar Raúl Mancilla Villa

Correo electrónico: oscar.mancilla@academicos.udg.mx

Recibido: 15 de mayo de 2020

Aceptado: 20 de octubre de 2021
Volumen XXIV, Número 1 
incluyendo las acciones antrópicas (Cotler et al., 2010; Geraldi et al., 2010; Báez, 2014).

En Jalisco, la demanda de aguas superficiales está ligada al crecimiento poblacional y el desarrollo de actividades productivas (CONAGUA, 2016). La cuenca del río Ayuquila-Armería, es la más grande en el estado de Colima y la segunda en Jalisco, está dentro de la clasificación de las cuencas hidrográficas prioritarias de México por su alta biodiversidad, pero a la vez por su grado de alteración de la dinámica funcional y los niveles altos de presión social que presenta (Cotler et al., 2010; Contreras-Rodríguez et al., 2020).

La compleja problemática que presenta se debe a la contaminación de cuerpos de agua por sedimentos en suspensión, las descargas de drenajes urbanos a los cuerpos de agua, la modificación del entorno por una fuerte deforestación, el abatimiento de acuíferos en la parte media y baja de la cuenca, el desvío y la retención del agua que ocasionan el desecamiento de ríos y arroyos, el crecimiento demográfico, el uso ineficiente del agua urbana y agrícola, las especies introducidas de tilapia, el uso inadecuado de redes de pesca, la cacería furtiva y el cultivo de estupefacientes (Santana et al., 1993; Mancilla-Villa et al., 2017; Meza et al., 2017).

Debido a la problemática y a la contaminación a la que se enfrenta el río, es necesario establecer un registro del análisis de la calidad del agua, haciendo énfasis sobre el aspecto de la contaminación orgánica. En este sentido, el presente estudio se enfoca en la evaluación de diversos parámetros de calidad del agua, considerando las concentraciones de nitratos, fosfatos, el contenido microbiológico y la aplicación de un índice de integridad biótica para evaluar el grado de contaminación orgánica. Los resultados se comparan con lineamientos nacionales (NOM-001-ECOL-1996.); además se complementa la información con la medición de parámetros fisicoquímicos como el pH, conductividad eléctrica (C.E.) y el caudal. Dándole un enfoque de importancia a las cuencas tributarias, las cuales dependiendo de su estado de calidad promueven el saneamiento o contaminación del cauce principal de la cuenca, se presentan recomendaciones para la mitigación de la contaminación hídrica.

\section{MATERIALES Y MÉTODOS \\ Área de estudio}

El río Ayuquila-Armería se localiza en los estados de Jalisco y Colima, en el occidente del país, entre las coordenadas geográficas $18^{\circ} 51^{\prime} 05^{\prime \prime}$ a $20^{\circ} 28^{\prime} 03^{\prime \prime} \mathrm{N}$ y $104^{\circ} 38^{\prime}$ 17 " a $103^{\circ} 34^{\prime} 41^{\prime \prime} \mathrm{O}$; cuenta con una extensión de 10257 $\mathrm{km}^{2}$; su origen por la confluencia de los ríos Ayuquila y del río Tuxcacuesco. Poco antes de ingresar al estado de Colima, toma el nombre de río Armería; recorre una distancia de 321 $\mathrm{km}$ desde la parte más alta (3290 msnm) hasta su desembocadura en el estero Boca de Pascuales en Colima (Cotler y Caire, 2009; Meza-Rodríguez et al., 2017).

La cuenca es clasificada con clima semiseco muy cálido, cálido subhúmedo, semicálido subhúmedo y templado subhúmedo, todos con lluvias en verano; temperatura media anual de $14-28{ }^{\circ} \mathrm{C}$; y una precipitación total anual de 700-
2000 mm, con evaporación del 80-90\% de la precipitación total (Mancilla-Villa et al., 2017). En cada sitio se determinaron las coordenadas geográficas mediante un GPS (Garmin, eTrex 10) (Figura 1).

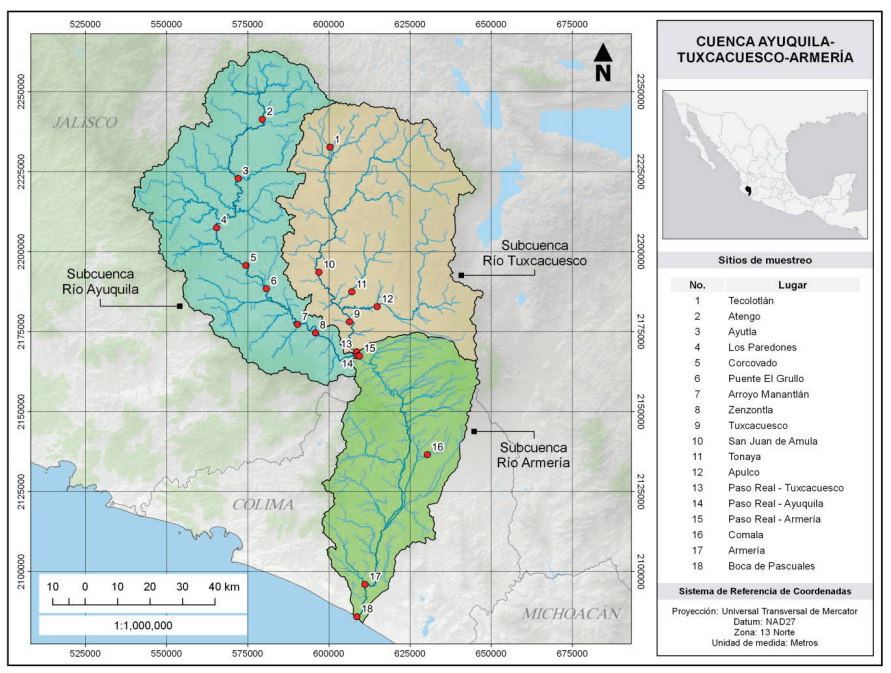

Figura 1. Localización geográfica de los sitios de muestreo en la cuenca del Río Ayuquila-Armería.

Figure 1. Geographical location of sampling sites at the Ayuquila-Armería River basin.

\section{Muestreo y análisis}

Se realizaron dos campañas de muestreos en la cuenca del Río Ayuquila-Armería y sus afluentes, abarcando la parte alta, media y baja de la misma, distribuyendo 18 sitios de colecta en la temporada de lluvia (octubre 2015) así como para la temporada seca (marzo 2016) (Figura 1). En cada sitio se colectaron muestras de agua $(500 \mathrm{~mL}$ ) y de macroinvertebrados acuáticos, aunado a esto se determinaron parámetros fisicoquímicos ( $\mathrm{pH}, \mathrm{C}$. E y caudal).

Las muestras de agua se obtuvieron en cada uno de los puntos de muestreo utilizando recipientes de polietileno de alta densidad de $0.2 \mathrm{~L}$, a cada envase se le colocó una etiqueta con su respectiva fecha, nombre y localización del sitio, posteriormente en laboratorio se determinó $\mathrm{pH}$, conductividad eléctrica, nitratos y fosfatos (Solís-Garza et al., 2011; Mancilla-Villa et al., 2017).

\section{Colecta de macroinvertebrados}

La colecta de los macroinvertebrados se realizó con una red tipo $D$ con una luz de malla de $600 \mu \mathrm{m}$, cubriendo las dos orillas del cauce y en dos muestreos en el centro para un total de $4 \mathrm{~m}^{2}$ por sitio, los organismos colectados fueron contados in situ hasta obtener 120 individuos, posteriormente se colocaron en recipientes de plástico con alcohol al $70 \%$ para ser transportados en hieleras e identificados en el laboratorio (Terneuz et al., 2012; Gutiérrez-Garaviz et al., 2014)

Los macroinvertebrados fueron agrupados como colector-filtrador, colector-recolector, raspadores, desmenuzadores y depredadores, de acuerdo con la clasificación de Merritt y Cummins (1996). Se calculó el Índice de ShannonWiener para conocer la diversidad de familias de cada sitio, 
además, se analizó la tolerancia a la contaminación orgánica de cada familia por medio del Índice de Integridad Biótica (IIB) siguiendo la metodología de Henne et al. (2002) y Weigel et al. (2002) (Tabla 1). Los valores del índice van de 0 a 10, donde 10 es indicativo de alta concentración de nutrientes, por lo tanto, las comunidades estarán compuestas de especies cuya tolerancia a la contaminación orgánica varía de sensible a tolerante. Una vez puntuadas, las clasificaciones de calidad varían de excelentes a muy pobres en función de la probable cantidad de contaminación orgánica en el sitio muestreado (Tabla 2).

Tabla 1. Lista de macroinvertebrados y su valor de tolerancia para el análisis del Índice de Integridad Biótico (IIB).

Table 1. List of macroinvertebrates and their tolerance value for the analysis of the biotic integrity index (IIB).

\begin{tabular}{|c|c|c|c|c|}
\hline Phylum & Clase/Orden & Familia & $\begin{array}{c}\text { Valor } \\
\text { IIB }\end{array}$ & $\begin{array}{c}\text { Grupo } \\
\text { funcional }\end{array}$ \\
\hline Platyhelminthes & Turberllaria & & 4 & $\mathrm{SH}$ \\
\hline \multirow{2}{*}{ Annelida } & Oligochaeta & & 8 & $\mathrm{SH}$ \\
\hline & Hirudinea & Hirudidae & 8 & $\mathrm{SH}$ \\
\hline \multirow{27}{*}{ Arthropoda } & \multirow{3}{*}{ Decapoda } & Cambaridae & 6 & $\mathrm{SH}$ \\
\hline & & Palaemonidae & 6 & $\mathrm{SH}$ \\
\hline & & Leptohyphidae & 4 & CG \\
\hline & \multirow[t]{3}{*}{ Ephemeroptera } & Leptophlebiidae & 2 & CG \\
\hline & & Baetidae & 4 & CG \\
\hline & & Gomphidae & 1 & PR \\
\hline & \multirow{3}{*}{ Odonata } & Libellulidae & 9 & PR \\
\hline & & Calopterygidae & 5 & PR \\
\hline & & Coenagrionidae & 9 & PR \\
\hline & Plecoptera & Perlidae & 1 & PR \\
\hline & \multirow{2}{*}{ Hemiptera } & Naucoridae & 5 & PR \\
\hline & & Belostomatidae & 10 & PR \\
\hline & \multirow[t]{2}{*}{ Megaloptera } & Corydalidae & 0 & PR \\
\hline & & Dryopidae & 5 & $\mathrm{SH}$ \\
\hline & \multirow{3}{*}{ Coleoptera } & Dytiscidae & 5 & PR \\
\hline & & Elmidae & 4 & CG \\
\hline & & Psephenidae & 4 & SC \\
\hline & \multirow[t]{2}{*}{ Lepidoptera } & Pyralidae & 5 & $\mathrm{SH}$ \\
\hline & & Hydropsychidae & 4 & $\mathrm{CF}$ \\
\hline & \multirow[t]{2}{*}{ Trichoptera } & Philopotamidae & 3 & $\mathrm{CF}$ \\
\hline & & $\begin{array}{l}\text { Polycentropodi- } \\
\text { dae }\end{array}$ & 6 & $\mathrm{CF}$ \\
\hline & \multirow{6}{*}{ Diptera } & Ceratopogonidae & 6 & PR \\
\hline & & Simuliidae & 6 & $\mathrm{CF}$ \\
\hline & & Tipulidae & 6 & CG \\
\hline & & Empididae & 6 & CG \\
\hline & & Tabanidae & 6 & PR \\
\hline & & Chironomidae & 6 & CG \\
\hline
\end{tabular}

Grupos funcionales: CF: Colector-filtrador; CG: Colector-recolector; SC: Raspadores; SH: Desmenuzadores; PR: Depredadores. Fuente: Merritt y Cummins (1996); Henne et al. (2001); Weigel et al. (2002).
Tabla 2. Parámetros utilizados para el Índice de Integridad Biótico (IIB). Table 2. Parameters used for the Biotic Integrity Index (IIB).

\begin{tabular}{|c|c|c|}
\hline IIIB & Calidad & Grado de contaminación \\
\hline $0.00-3.75$ & Excelente & $\begin{array}{l}\text { Ninguna contaminación orgánica } \\
\text { aparente }\end{array}$ \\
\hline $3.76-4.25$ & Muy buena & Posible contaminación orgánica \\
\hline $4.26-5.00$ & Buena & Con alguna contaminación orgánica \\
\hline $5.01-5.75$ & Media & $\begin{array}{c}\text { Contaminación orgánica bastante } \\
\text { significativa }\end{array}$ \\
\hline $5.76-6.50$ & $\begin{array}{l}\text { Bastante } \\
\text { pobre }\end{array}$ & Contaminación orgánica significativa \\
\hline $6.51-7.25$ & Pobre & $\begin{array}{c}\text { Contaminación orgánica muy } \\
\text { significativa }\end{array}$ \\
\hline $7.26-10.0$ & Muy pobre & Contaminación orgánica severa \\
\hline
\end{tabular}

Fuente: Henne et al. (2002).

\section{Análisis microbiológico}

El estudio bacteriológico en el agua de las microcuencas y el cauce principal se desarrolló de la forma siguiente: en frascos de polietileno de $500 \mathrm{~mL}$, previamente lavados y esterilizados, se colectaron las muestras de agua de cada sitio; en su interior, previo a la esterilización, se colocó 0.1 $\mathrm{cm}^{3}$ de solución de disulfato de sodio al $1 \%$ con el propósito de inhibir la acción del cloro que puede contener la muestra, cubriendo el tapón del frasco con papel aluminio como establece la NMX-AA-42 (1987) (Mora-Bueno et al., 2012).

Las muestras de agua se llevaron al Laboratorio de Investigación en Biotecnología del Centro Universitario de la Costa Sur (CUCSUR) de la Universidad de Guadalajara para la realización de cultivos sobre placas Petrifilm EC para el recuento de E. coli / Coliformes que contiene nutrientes de Bilis Rojo Violeta (VRB), un agente gelificante soluble en agua fría, un indicador de actividad de la glucuronidasa y un indicador que facilita la enumeración de las colonias (Pucci et al., 2013).

Sobre las placas Petrifilm se coloca $1 \mathrm{~mL}$ de muestra con pipetas esterilizadas, posteriormente se esparció uniformemente la muestra sobre la placa. Después de incubar las placas 24 hrs a $35^{\circ} \mathrm{C}$ según método 991.14 del AOAC (2002), se contabilizan los puntos: los puntos azules son Unidades Formadoras de Colonias (UFC) de E. coli y los puntos rojos son colonias de coliformes fecales, que al sumarlas resulta en el total de colonias de coliformes (Redondo y Arias, 2011).

\section{Estimación del caudal}

Para la estimación del caudal se utilizó el método de las huellas máximas (Elosegui y Sabater, 2009) para cuantificar los volúmenes de aporte de las principales cuencas tributarias y del cauce principal. Por medio de este método se estimó el gasto máximo que se presenta durante una avenida reciente en un río donde no se cuenta con ningún otro tipo de aforo (Martínez y Fernández 2010). En la ecuación utilizada se tiene que: 


\section{$\mathrm{Q}=\mathrm{V}^{*} \mathrm{~A}$}

Dónde: $\mathrm{Q}=$ Gasto de la avenida $\left(\mathrm{m}^{3} / \mathrm{s}\right) \mathrm{A}=$ Área hidráulica en $\mathrm{m}^{2} . V=$ Velocidad $(\mathrm{m} / \mathrm{s})$.

Para obtener el área hidráulica, en cada punto de muestreo, se midió la profundidad que alcanza el cauce del río junto con la velocidad del caudal en cada metro a través del ancho total del caudal, una vez obtenidos estos dos parámetros se estimaron los volúmenes de aporte de cada sitio (De la Lanza et al., 2012).

\section{Análisis estadístico}

Los resultados se sometieron a un análisis estadístico para conocer la correlación entre muestreos realizados, se llevó a cabo análisis de varianza (ANOVA) para determinar las diferencias significativas entre éstos. Para realizar el análisis estadístico se utilizó el programa Stadistical Package for the Social Sciences (SPSS) versión 25.0. Adicionalmente se realizó un Análisis de Correspondencia canónica (ACC), para conocer la relación entre las variables fisicoquímicas del agua ( $\mathrm{pH}, \mathrm{C}$.E y Caudal) y la presencia o ausencia de las familias de macroinvertebrados acuáticos. Para realizar este análisis se utilizó el software PC ORD V 6.0.

\section{RESULTADOS Y DISCUSIÓN Variables fisicoquímicas}

El valor de $\mathrm{pH}$ en la temporada lluviosa fluctuó entre 7.34 - 8.43 con un promedio de 7.98; en la temporada seca el valor fue entre 7.35 - 8.39 con un promedio de 7.84 (tabla 3); no se encontraron diferencias significativas entre las temporadas de muestreo $(F=1.51 ; p=0.22$ ) (tabla 4). Estos valores se consideran alcalinos (Díaz et al., 2018) y se encuentran dentro del rango permisible (5 a 10 unidades) que establece la NOM-001-ECOL-1996, en su apartado de protección a la vida acuática. No obstante, se ha evidenciado que para utilizar el agua en el riego agrícola se recomiendan valores entre 5.5 y 6.5 , ya que la mayoría de los iones se encontraran disponibles para la planta (Ortiz-vega et al., 2019). Los valores de pH encontrados coinciden con los reportados en otros estudios realizados en la cuenca del río Ayuquila-Armería (MancillaVilla et al., 2017), y en otras regiones del país (Guzmán-Colis et al., 2011) lo que indica que existe poca variación temporal en cuanto al $\mathrm{pH}$.

Los valores de C.E. fluctuaron de 137 a $2225 \mu \mathrm{S} / \mathrm{cm}$ en ambas campañas de colecta, los valores de 2225 y 2123 $\mu \mathrm{S} / \mathrm{cm}$ corresponden al sitio de Boca de Pascuales, punto de desembocadura en el mar y es donde se combina el flujo de agua salada con la del río (tabla 3); no se encontraron diferencias significativas entre temporadas de muestreo $(F=0.21 ; p=$ 0.64) (tabla 4).

En este sentido los valores encontrados representan riesgo para la vida acuática en organismos de agua dulce en los sitios donde se superan los $1000 \mu \mathrm{S} / \mathrm{cm}$, ya que se verían afectadas algunas de sus funciones vitales como la reproducción en algunas especies de peces (Meza y Sepúlveda, 2012). En cuanto al aspecto de riego agrícola estos valores representan riesgo en cuanto la utilización del recurso hídri-
Tabla 3. Parámetros fisicoquímicos determinados en los sitios de muestreo. Table 3. Physicochemical parameters determined at sampling sites.

\begin{tabular}{|c|c|c|c|c|c|c|}
\hline \multirow{2}{*}{$\begin{array}{c}\text { Sitio } \\
\begin{array}{c}\text { Estación } \\
\text { (n) }\end{array}\end{array}$} & \multicolumn{2}{|c|}{ pH } & \multicolumn{2}{|c|}{ C.E. $(\mu \mathrm{S} / \mathrm{cm})$} & \multicolumn{2}{|c|}{ Caudal $\left(\mathrm{m}^{3} / \mathrm{s}\right)$} \\
\hline & Lluviosa & Seca & Lluviosa & Seca & Lluviosa & Seca \\
\hline 1 & 7.88 & 7.72 & 122 & 137 & 0.17 & 0.03 \\
\hline 2 & 7.34 & 7.35 & 158 & 149 & 1.24 & 0.44 \\
\hline 3 & 7.79 & 7.59 & 182 & 187 & 3.67 & 1.02 \\
\hline 4 & 7.7 & 7.61 & 155 & 143 & 26.96 & 16.14 \\
\hline 5 & 7.9 & 7.87 & 162 & 162 & 27.03 & 16.32 \\
\hline 6 & 7.59 & 7.42 & 172 & 182 & 8.29 & 3.82 \\
\hline 7 & 7.48 & 7.46 & 56 & 112 & 4.13 & 1.37 \\
\hline 8 & 8.18 & 8.29 & 195 & 221 & 43.41 & 14.46 \\
\hline 9 & 8.11 & 8.12 & 419 & 423 & 32.89 & 11.49 \\
\hline 10 & 7.77 & 7.43 & 333 & 347 & 5.56 & 1.80 \\
\hline 11 & 8.44 & 8.28 & 504 & 513 & 0.34 & 0.03 \\
\hline 12 & 8.43 & 8.41 & 235 & 246 & 0.29 & 0.03 \\
\hline 13 & 8.07 & 8.01 & 432 & 427 & 30.08 & 9.37 \\
\hline 14 & 7.99 & 7.55 & 286 & 279 & 45.19 & 19.99 \\
\hline 15 & 8.04 & 7.71 & 379 & 385 & 75.26 & 29.67 \\
\hline 16 & 8.12 & 7.99 & 233 & 244 & 1.07 & 0.29 \\
\hline 17 & 8.4 & 7.87 & 573 & 561 & 59.06 & 18.40 \\
\hline 18 & 8.35 & 8.39 & 2225 & 2123 & 58.34 & 16.93 \\
\hline Promedio & 7.98 & 7.84 & 378 & 380 & 23.50 & 8.98 \\
\hline Desv Std & 0.326 & 0.353 & 482 & 455 & 24.40 & 9.18 \\
\hline
\end{tabular}

co, ya que los valores de restricción se establecen al superar los $1000 \mu \mathrm{S} / \mathrm{cm}$ (Can-Chulim et al., 2014). Se ha evidenciado que valores que exceden los $1500 \mu \mathrm{S} / \mathrm{cm}$ presentan efectos negativos sobre la germinación de algunas semillas, como el maíz (Zea Mays) donde el porcentaje de germinación de las semillas disminuyo al utilizar aguas con una C.E. por arriba de los valores antes mencionados (Fessel et al., 2006; Viloria et al., 2011).

En cuanto a los resultados de la medición del caudal, en la primera campaña de muestreo las determinaciones variaron de 0.18 a $75.27 \mathrm{~m}^{3} / \mathrm{s}$ con un promedio de 23.50 $\mathrm{m}^{3} / \mathrm{s}$. En la segunda campaña los valores oscilaron entre 0.03 a $29.68 \mathrm{~m}^{3} / \mathrm{s}$ con un promedio de $8.98 \mathrm{~m}^{3} / \mathrm{s}$. Para este parámetro sí se encontraron diferencias significativas entre las temporadas de muestreo $(\mathrm{F}=5.58 ; \mathrm{p}=0.024)$ (tabla 4$)$. Los valores más altos ( $>15 \mathrm{~m}^{3} / \mathrm{s}$ ) corresponden a las mediciones realizadas en los cauces principales, y los valores más bajos $\left(<15 \mathrm{~m}^{3} / \mathrm{s}\right)$ corresponden a las mediciones realizadas en los afluentes del mismo cauce.

El caudal del río se considera como un factor muy importante ya que este influye directamente en la variación de los niveles de parámetros fisicoquímicos y en las concentraciones de contaminantes orgánicos (Guevara et al., 2006; Encalada, 2010). La relación del caudal con la concentración de algunos contaminantes se ha puesto en evidencia en estudios como el de Gaete et al. (2007), en el cual se demostró que los niveles de contaminantes; coliformes y Nitrógeno, 
Tabla 4. Análisis de varianza (ANOVA) para ambas campañas de muestreo.

Table 4. Analysis of variance (ANOVA) for both sampling campaigns.

\begin{tabular}{|c|c|c|c|c|c|c|}
\hline Determinaciones & & $\begin{array}{l}\text { Suma de } \\
\text { cuadrados }\end{array}$ & Gl & $\begin{array}{l}\text { Media } \\
\text { cuadrática }\end{array}$ & $\mathbf{F}$ & p sig. \\
\hline \multirow{3}{*}{$\mathrm{pH}$} & Entre grupos & 0.175 & 1 & 0.17 & 1.51 & 0.22 \\
\hline & Dentro de grupos & 3.91 & 34 & 0.11 & & \\
\hline & Total & 4.09 & 35 & & & \\
\hline \multirow{3}{*}{ C.E. } & Entre grupos & 0.04 & 1 & 0.004 & 0.21 & 0.64 \\
\hline & Dentro de grupos & 0.68 & 34 & 0.20 & & \\
\hline & Total & 0.69 & 35 & & & \\
\hline \multirow{3}{*}{ Caudal } & Entre grupos & 1897.69 & 1 & 1897.69 & 5.58 & $0.024^{*}$ \\
\hline & Dentro de grupos & 11551.74 & 34 & 339.75 & & \\
\hline & Total & 13449.43 & 35 & & & \\
\hline \multirow{3}{*}{ UFC } & Entre grupos & 7453.44 & 1 & 7453.44 & 4.36 & $0.044^{*}$ \\
\hline & Dentro de grupos & 58112.44 & 34 & 1709.19 & & \\
\hline & Total & 65565.88 & 35 & & & \\
\hline \multirow{3}{*}{$P_{\text {Total }}$} & Entre grupos & 0.019 & 1 & 0.019 & 0.11 & 0.73 \\
\hline & Dentro de grupos & 5.55 & 34 & 0.16 & & \\
\hline & Total & 5.57 & 35 & & & \\
\hline \multirow{3}{*}{$\mathrm{N}_{\text {Total }}$} & Entre grupos & 647.70 & 1 & 647.70 & 1.04 & $0.031^{*}$ \\
\hline & Dentro de grupos & 21118 & 34 & 321.12 & & \\
\hline & Total & 21766 & 35 & & & \\
\hline
\end{tabular}

Diferencia significativa $(\mathrm{p} \leq 0.05)$ *

disminuían cuando el caudal en el Río Aconcagua llegaba a sus puntos máximos y al contrario se encontraron mayores concentraciones de estos en los meses donde el caudal disminuía. En otro estudio en el río Condoto en Colombia, se evidenció que las concentraciones de contaminantes provenientes de las zonas urbanas y mineras disminuían cuando por la estacionalidad aumentaba el caudal del río, y en contraparte se encontraron mayores concentraciones de contaminantes cuando el caudal disminuía (Sánchez y Cañón, 2010).

Las concentraciones de fósforo total $\left(\mathrm{P}_{\text {Tot }}\right)$ se clasificaron de acuerdo con los valores de peligro de eutrofización de cuerpos de agua propuesta por Sharpley (2010), la cual indica que una concentración mayor a $0.1 \mathrm{mg} / \mathrm{L}$ de $\mathrm{P}$ disuelto puede causar la eutrofización acelerada de las aguas.

En la figura 2, se muestra la concentración de $\mathrm{P}_{\text {Total }}$ de las aguas superficiales en ambas campañas de muestreo y el límite que representa el peligro de eutrofización acelerada. No se encontró variación en la concentración de $\mathrm{P}$ en las dos temporadas, excepto en El Corcovado, donde se presentó en temporada lluviosa una concentración de 0.01 y en temporada seca una concentración de $0.48 \mathrm{mg} \mathrm{L}^{-1}$.

Fregoso-Zamorano (2015) en la misma zona de estudio registró una concentración de $0.02 \mathrm{mg} / \mathrm{L}$ de $\mathrm{P}$ en El Corcovado en el mes de octubre del 2013, valor que aumentó a $0.5 \mathrm{mg} / \mathrm{L}$ en marzo del 2014, demostrando que existe una constante de que en la temporada lluviosa la concentración en El Corcovado disminuye para después aumentar en la temporada seca. En general, las aguas del río Ayuquila-
Tuxcacuesco-Armería contienen poca cantidad de fósforo que pueden aportar a los cultivos sin ocasionar problemas, pero en la mayoría de los sitios el fósforo contenido acelera el proceso de eutrofización en las aguas. Las actividades antropogénicas, como la utilización de grandes cantidades de detergentes y fertilizantes, aportan fósforo a las aguas superficiales y aceleran este proceso (Neal et al., 2006).

Se han realizado otros estudios referentes a la determinación de fósforo y sus diferentes formas en aguas superficiales y subterráneas. Tal es el caso del estudio desarrollado en el sistema hidrográfico Zahuapan-Atoyac por Carrera-Villacrés et al. (2011), donde se encontró que las concentraciones de $\mathrm{P}$ y $\mathrm{PO}_{4}$, en los orígenes de los ríos Zahuapan y Atoyac son

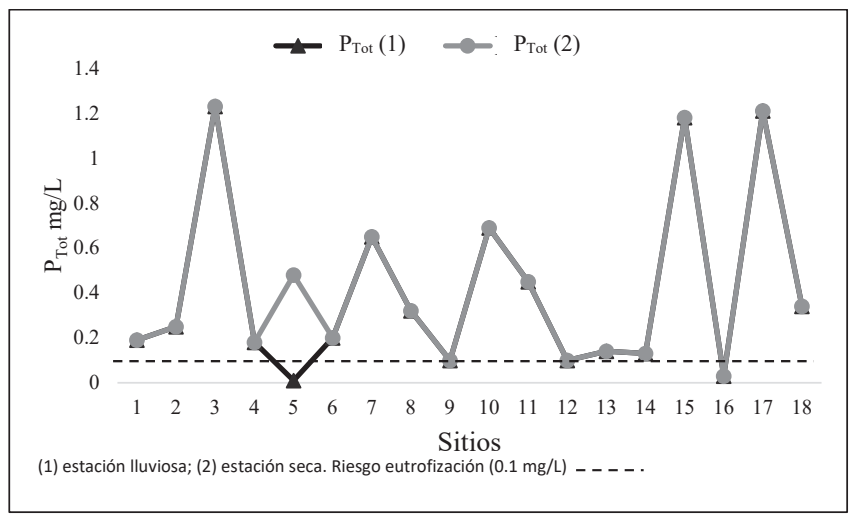

Figura 2. Concentración de $P_{\text {Total }}$ en los sitios muestreados en la estación lluviosa y seca.

Figure 2. $\mathrm{P}_{\text {Total }}$ concentration at sampled sites in the rainy and dry season. 
bajas, de 0.3 a $0.5 \mathrm{mg} / \mathrm{L}$. En cambio, cuando los cauces de estos ríos ingresan por poblados urbano-industriales, las concentraciones aumentan considerablemente y varían de 1.0 a $7.89 \mathrm{mg} / \mathrm{L}$. Los resultados se asemejan a los encontrados en el presente estudio y se infiere que esta semejanza es el resultado de la influencia de las condiciones ambientales que atraviesa el río Ayuquila-Armería, el cual transita por zonas industriales y urbanas, donde las aguas residuales no reciben tratamiento alguno.

En la figura 3, se presentan las concentraciones de $\mathrm{N}_{\text {Total }}$ en ambas campañas de muestreo (estación lluviosa y seca) y su comparación con el límite permisible de concentración relativo a la protección de la vida acuática $(25 \mathrm{mg} / \mathrm{L}$ ) propuesto por la NOM-001-ECOL-1996. Se encontraron diferencias significativas entre temporadas de muestreo $(F=1.04 ; p=0.031)$ (tabla 4). El $50 \%$ de los sitios muestreados supera el límite permisible, las concentraciones más altas se encontraron en la temporada lluviosa en los sitios 4 y 18.

Las variaciones de concentración de $\mathrm{N}_{\text {Total }}$ en el muestreo de la temporada lluviosa y seca, encontradas en las aguas de los sitos muestreados se relacionan con la descarga de aguas residuales agrícolas, que derivan de la aplicación de fertilizantes nitrogenados, así como de las descargas de aguas residuales provenientes de granjas porcícolas y aguas residuales urbanas sin tratamiento como se ha evidenciado en otros trabajos de investigación realizados en la región de estudio (Palomera-García, 2012; Mancilla-Villa et al., 2017).

En un estudio realizado en el río Zahuapan, México por Muñoz-Nava et al. (2013) encontraron que los promedios de concentración de $\mathrm{N}_{\text {Total }}$ en las doce microcuencas estudiadas no fueron mayores a $15 \mathrm{mg} / \mathrm{L}$, la concentración más elevada fue de $11.5 \mathrm{mg} / \mathrm{L}$; estos resultados se atribuyen a un posible efecto de la presa de Atlangatepec y a la capacidad de retención de nutrientes de la vegetación acuática que se encuentra en el cauce del río. Los resultados encontrados difieren a los del presente estudio. En base a los estudios realizados se puede inferir que la vegetación ribereña tiene un papel muy importante en cuanto a la retención de contaminantes (Rodríguez-Contreras et al., 2020), situación que se ve reflejada el estado de la condicione ecológica de las riberas de la cuenca del río Ayquila-Armeria.

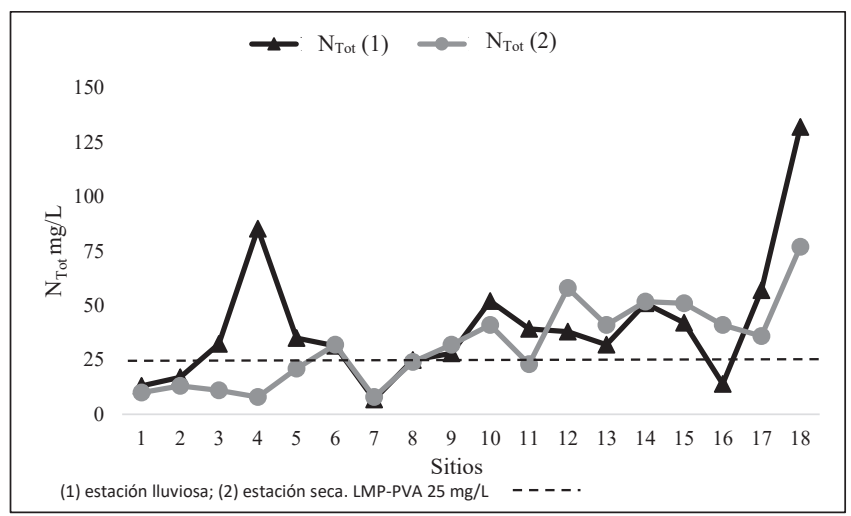

Figura 3. Concentración de $\mathrm{N}_{\text {Total }}$ en los sitios muestreados en la estación lluviosa y seca.

Figure 3. $\mathrm{N}_{\text {Total }}$ concentration at sampled sites in the rainy and dry season.

\section{Índice de integridad biótica}

Se colectaron e identificaron un total de 2634 macroinvertebrados, 1878 en temporada lluviosa pertenecientes a 20 familias, y 756 en temporada seca pertenecientes a 20 familias, en 18 sitios del área de estudio (figura 4). La familia más abundante fue Leptopheibidae con 729 individuos colectados en las dos temporadas, le sigue la familia Hydropsychidae con 630 individuos. En general se colectaron más especímenes en la temporada lluviosa que en la temporada seca, esto coincide con lo reportado en otros estudios en la zona (Palomera-García, 2012; Hernández-Vargas et al., 2017).

En cuanto a la diversidad en los sitios, los valores de diversidad varían de 0.04 a 2.20 en ambas temporadas (figura 5), que coincide con el rango registrado por Palomera-García (2012) en un estudio realizado en la cuenca del río Ayuquila - Tuxcacuesco - Armería, donde registró un rango de diversidad de 0.19 a 2.19. El sitio con mayor diversidad $\left(\mathrm{H}^{\prime}\right)$ fue Arroyo Manantlán (sitio 7) en las dos temporadas muestreadas (2.20 en temporada lluviosa y 2.12 en temporada seca), sin embargo, en general no existe un patrón en los sitios que indique en cual temporada aumenta o disminuye la diversidad.

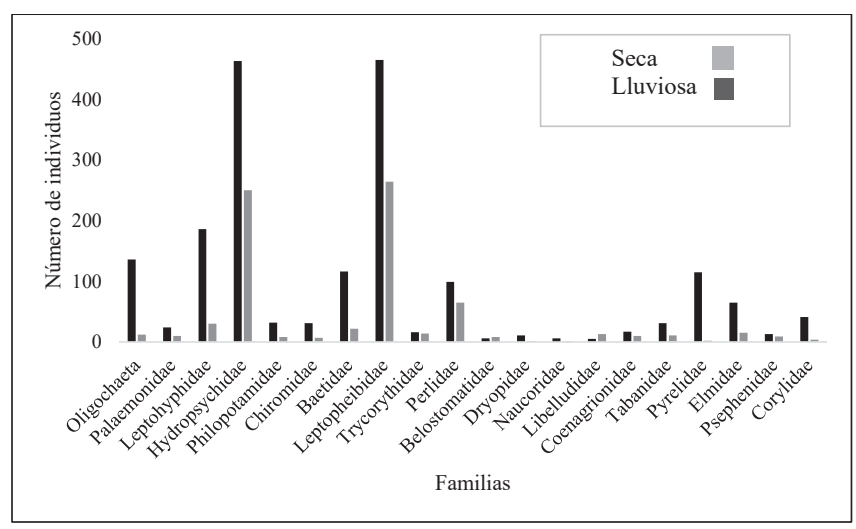

Figura 4. Número de individuos colectados por familia en ambas temporadas de muestreo.

Figure 4. Number of individuals collected per family at both sampling seasons.

En la figura 5 se presentan los resultados obtenidos de la aplicación del índice de integridad biótica (IIB). En la temporada de lluvias, de acuerdo con Henne et al. (2002), los sitios con calidad excelente $(\mathrm{IIB}<3.5)$ sin ninguna contaminación orgánica aparente, representaron el $69 \%$ de los sitios. No hay sitios con buena calidad, otro $6 \%$ corresponde a sitios de calidad buena, otro $6 \%$ es de calidad media y le siguen los sitios de calidad pobre con un $13 \%$ y por último los sitios de calidad muy pobre (IIB > 6.5) representan el $6 \%$ del total.

Los sitios con calidad excelente en la temporada seca representaron el $47 \%$; el $29 \%$ de los sitios fueron de calidad muy buena; los sitios de calidad buena representaron el 18 $\%$; le siguieron los sitios de calidad media con un $6 \%$ del total; no se presentaron sitios con calidad pobre o muy pobre.

En general, hay una mayor cantidad de sitios con calidad excelente en la temporada lluviosa que en la seca, no obstante, también hay más sitios con calidad pobre o muy 


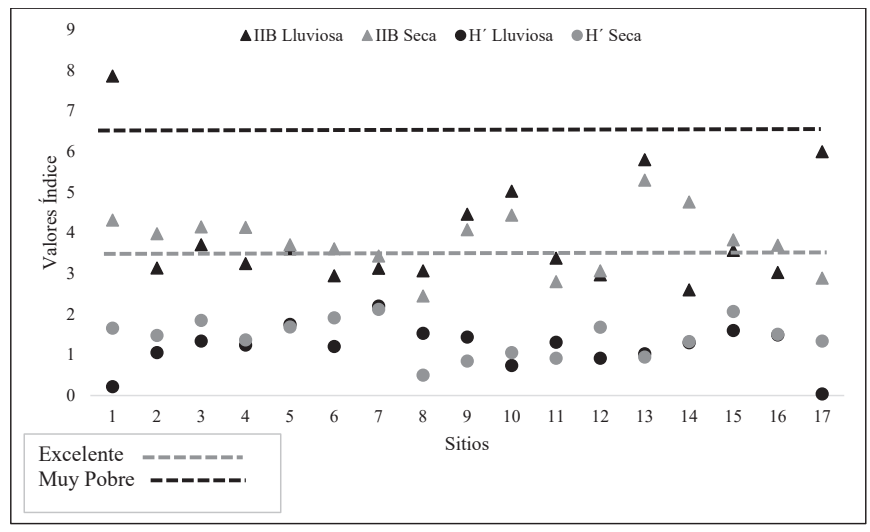

Figura 5. Índices de integridad biótica y diversidad por temporada de muestreo. IIB= índice de integridad biótica, $\mathrm{H}^{\prime}=$ índice de Shannon-Wiener. Figure 5. Biotic integrity and diversity indices per sampling season. $I I B=$ biotic integrity index, $\mathrm{H}^{\prime}=$ Shannon-Wiener index.

pobre en esta misma temporada. Los resultados concuerdan con lo registrado por Palomera-García (2012), quien registró una mayor cantidad de sitios de excelente y muy buena calidad de acuerdo con su IIB en la temporada de lluvias.

El análisis de correspondencia canónica (figura 6), indica que la varianza acumulada de la relación entre la presencia de las comunidades biológicas y las variables ambientales seleccionadas se explica en los dos primeros ejes con un 63 $\%$. En aquellos sitios con los valores más altos de caudal, se encuentra la familia Perlidae, esta familia de macroinvertebrados tiene una relación positiva con el caudal y se considera una de las familias con mayor grado de sensibilidad a la contaminación orgánica (Merrit y Cummins 1996).

Por su parte la variable de $P_{\text {Total }}$ presenta una relación positiva sobre la familia Leptopheibidae, los valores de $P_{\text {Total }}$ fueron bastante homogéneos entre las dos temporadas y no existe alta variabilidad entre sitios. La variable de $\mathrm{N}_{\text {Total }}$ tiene relación con las familias Baetidae e Hydropsychidae, estas tienen mayores facilidades de adaptación y por lo tanto son los más tolerantes a efectos de condiciones extremas o de perturbación (Moya et al., 2011; Roldan-Pérez, 2016). Se puede apreciar mediante este análisis de correspondencia, que las comunidades biológicas se están separando entre sitios y entre temporadas de muestreo, lo anterior es el resultado de la misma estructura de las comunidades de macroinvertebrados y la correlación con las variables ambientales.

\section{Análisis microbiológico}

En la figura 7 se presenta la cuantificación de UFC en los sitios del Río Ayuquila y sus afluentes por temporada de muestreo. Estos resultados se comparan con el límite permisible (10 UFC/mL) propuesto por la NOM-001-ECOL-1996. El $50 \%$ de los sitios exceden el límite permisible en la estación Iluviosa, con concentraciones que van desde los $18 \mathrm{UFC} / \mathrm{mL}$ hasta $190 \mathrm{UFC} / \mathrm{mL}$; por otra parte, en la estación seca el $22 \%$ de los sitios superan el límite permisible, con concentraciones que oscilan entre 11 y $21 \mathrm{UFC} / \mathrm{mL}$. Se encontraron diferencias significativas entre las temporadas de muestreo $(F=4.36: p=$ 0.044) (tabla 4).

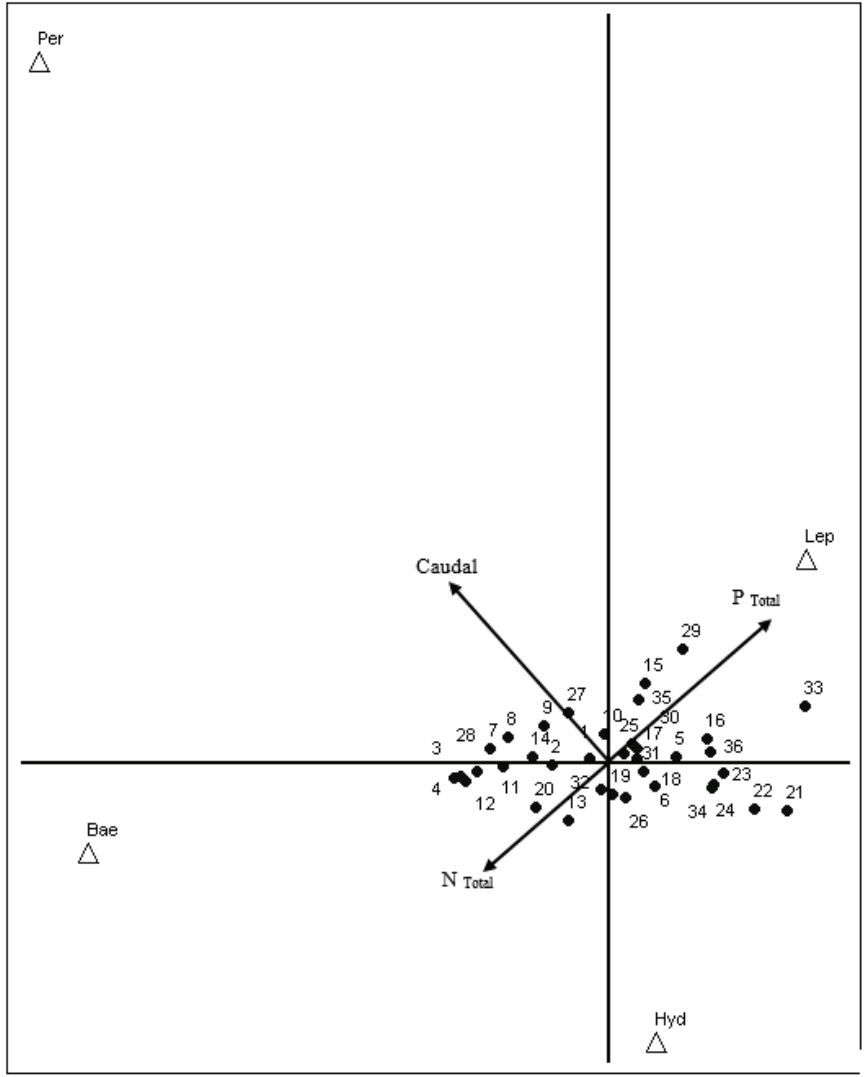

Figura 6. Análisis de correspondencia canónica en el ordenamiento de los sitios muestreados en ambas temporadas, relacionado con la presencia y abundancia de las familias de macroinvertebrados [Leptopheibidae (Lep), Baetidae (Bae), Perlidae (Per) e Hydropsychidae (Hyd)], colectados por sitio y correlacionado con las variables ambientales $\left(\mathrm{P}_{\text {Total' }} \mathrm{N}_{\text {Total }}\right.$ y caudal).

Figure 6. Canonical correspondence analysis in the sampled sites ordering in both seasons, related to the presence and abundance of the macroinvertebrate families [Leptopheibidae (Lep), Baetidae (Bae), Perlidae (Per) and Hydropsychidae (Hyd)], collected by site and correlated with environmental variables ( $\mathrm{P}_{\text {Total’ }} \mathrm{N}_{\text {Total }}$ and caudal).

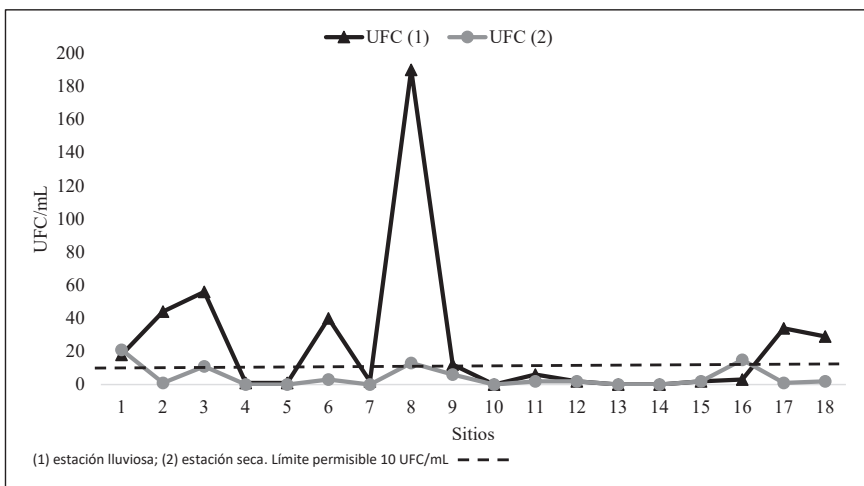

Figura 7. Concentración de unidades formadoras de colonias (UFC/mL) por temporada en los sitios muestreados.

Figure 7. Colony forming units concentration (CFU / $\mathrm{mL}$ ) per season at the sampled sites. 
El arrastre de residuos por la acción de la lluvia eleva las concentraciones de coliformes en la mayoría de los sitios, situación que se ve reflejada al encontrarse mayores concentraciones de UFC en la temporada lluviosa, esto se ha evidenciado en otros estudios (Ramírez et al., 2009; Olguín et al., 2010). Por otra parte, se les suman a las descargas provenientes de granjas porcícolas, las descargas de aguas residuales urbanas, tomando como referencia que en México el $70 \%$ de los municipios cuentan con planta de tratamiento de agua residual, y de estas, alrededor del $50 \%$ se encuentre en funcionamiento actualmente (CONAGUA, 2016).

El sitio que registró la mayor concentración de UFC fue el número 8 en la temporada de lluvias. Se infiere que estas concentraciones se deben a que, en este punto del río se vierten las aguas residuales de los municipios de Autlán y El Grullo, así como todo el arrastre de las lluvias del suelo agrícola del valle Autlán - El Grullo. En general las concentraciones aumentan en todos los sitios en la temporada de lluvias, debido a la descarga de aguas negras de áreas urbanas y drenaje agrícola, lo que sugiere una alta y peligrosa contaminación por patógenos intestinales (Robles et al., 2013; Anduro et al., 2017).

Por otro lado, los sitios 4 y 10, son sitios que se encuentran poco después de dos presas importantes en la región (Trigomil y Las Piedras), lo que sugiere una mayor dilución del agua y, por tanto, menos concentración de coliformes (Sardiñas et al., 2006). El caso del sitio 7 corresponde al Arroyo Manantlán, este es un afluente el cual tiene su nacimiento en la parte alta de la Reserva de la Biosfera Sierra de Manantlán, por ende, este afluente sufre muy poca o nula presión y contaminación urbana, por lo tanto, se considera como un sitio de referencia de buena calidad microbiológica (Santana et al., 1993).

Un estudio realizado por Olivas-Enríquez et al. (2011) en el Río Bravo determinó la concentración de coliformes totales y E. coli. La presencia indiscutible de enteropatógenos en el agua del Río Bravo constituye un riesgo para la salud pública, principalmente para los agricultores que la manejan, y en el riego por la contaminación de los productos agrícolas de consumo humano, así como para las personas que entran en contacto con el agua del río.

\section{CONCLUSIONES}

Se confirmó que existe alto riesgo de eutrofización en los cuerpos de agua analizados debido a las altas concentraciones de Fósforo total $\left(\mathrm{P}_{\text {Total }}\right)$ y Nitrógeno Total $\left(\mathrm{N}_{\text {Tota }} \mathrm{I}\right)$. Esto sugiere tomar acciones preventivas y correctivas en la disminución de la contaminación por estos elementos y analizar la supervisión de las actividades antropogénicas que emanan contaminantes fosfóricos y nitrogenados. El índice de integridad biótica mostró alta contaminación orgánica en $20 \%$ de los sitios en estudio, es un porcentaje relevante debido a que las actividades antropogénicas son las causas principales de esta problemática. Se demuestra que urge un plan estratégico a nivel cuenca para evitar los derrames, vertederos o lixiviados de contaminantes orgánicos. Estos hallazgos explican la problemática que debe atenderse con estrategias que generen alternativas de manejo de recursos hídricos e insumos agropecuarios con enfoques sustentables y que eviten descargas y arrastres de bacterias peligrosas para la salud humana y ecosistémica.

\section{REFERENCIAS}

Anduro, J., Cantú, S y Campos B. 2017. Diagnóstico de la calidad sanitaria del agua de pozo en comunidades del sur de Sonora, México. Revista Salud Publica y Nutrición. 16(1):1-8.

AOAC. 2002. AOAC Official method 991.14 coliform and Escherichia coli counts in foods. AOAC International. Washington, D.C.

Báez, W. 2014. Análisis del manejo de cuencas como herramienta para el aprovechamiento sustentable de recursos naturales. Revista Chapingo, Serie Zonas Áridas. 13(2): 39-45.

Can-Chulim, A., Ortega-Escobar, H. M., Sánchez-Bernal, E. I., y Cruz-Crespo, E. 2014. Calidad de agua para riego en la Sierra Norte de Puebla, México. Tecnologías y Ciencias del Agua. 5(5): 77-96.

Castro, M., Almeida, J y Díaz, D. 2014. Indicadores de la calidad del agua: evolución y tendencias a nivel global. Ingeniería solidara. 10(17): 111-124.

Castillo, O. y Vera, R. 2016. Agua potable y saneamiento en la nueva ruralidad de América Latina. Serie Agua para el desarrollo, Bogotá-Colombia, 500 pp.

Carrera-Villacrés, D., Ortega-Escobar, H., Ramírez-García, J., Ramírez-Ayala, C., Trejo-López, C y Becerril-Román, A. 2011. Boro, fosfatos e índices de salinidad del sistema hidrográfico Zahuapan-Atoyac, México. Tecnología y ciencias del agua. 2(2): 05-21.

Díaz, R., Duarte, H., Pallanez, M., Moreno, J., Mejia, J y Durazo, F. 2018. Análisis de los criterios para proteger la vida acuática: el río Sonora después del derrame minero de 2014. AquaLAC. 10 (1): 75-87.

CONAGUA. 2016. Estadísticas del agua en México. Secretaria de Medio Ambiente y Recursos Naturales. Ciudad de México, México, 282 pp.

Cotler, H. y Caire, G. 2009. Lecciones aprendidas del manejo de cuencas en México. Instituto Nacional de Ecología (INE), Fundación Gonzalo Río Arronte, I.A.P., WWF, la Organización Mundial de Conservación.

Cotler, H., A. Garrido, V. Bunge y M. L. Cuevas. 2010. Las cuencas hidrográficas de México: priorización y toma de decisiones. En Cotler A. H. 2010. Las cuencas hidrográficas de México. Diagnóstico y priorización. Instituto Nacional de Ecología y Cambio Climático. 120 pp.

Contreras-Rodríguez, F., Martínez-Rivera, L. y Ortiz-Arrona, C. 2020. Nivel de alteración ecohidrológica en ríos perennes de la cuenca del río Ayuquila-Armería. Biotecnia. 22(2): 28-38.

De la Lanza, G., Carbajal, J., Salinas, S y Barrios, J. 2012. Medición del caudal ecológico del río Acaponeta, Nayarit, comparando distintos intervalos de tiempo. Investigaciones geográficas. 78: 62-74.

Elósegui, A. y Sabater, S. 2009. Conceptos y técnicas en ecología fluvial. Boletín de la Real Sociedad de Geografía. 145: 374375.

Encalada, A. 2010. Funciones ecosistémicas y diversidad de los ríos: Reflexiones sobre el concepto de caudal ecológico y su aplicación en el Ecuador. Revista Polémika. 2(5): 40-47. 
Fessel, S., Vieira, R., Cruz, M y Panobianco, M. 2006. Pruebas de conductividad eléctrica de semillas de maíz según la temperatura y el período de almacenamiento. Pesquisa Agropecuaria Brasileira. 41 (10), 1551-1559.

Fregoso-Zamorano, B. 2015. Calidad de las aguas de riego de la cuenca del río Ayuquila-Tuxcacuesco-Armería. (Tesis de Licenciatura). Universidad de Guadalajara. Autlán de Navarro, Jalisco, México. 136 pp.

Gaete, H. F., Aranguiz, G., Cienfuegos y M. Tejos. 2007. Metales pesados y toxicidad de aguas del río Aconcagua en Chile. Química Nova. 30: 885-891.

Geraldi, A., Piccolo, M. y Perillo, G. 2010. Delimitación y estudio de cuencas hidrográficas con modelos hidrológicos. Investigaciones geográficas. 52: 215-225.

Guevara, E. 2006. Análisis de frecuencia y riesgo en hidrología. Manuscrito Dirección General de Postgrado, Universidad de Carabobo.

Gutiérrez-Garaviz, J., Zamora, H. y Andrade, S. 2014. Efecto de la actividad antrópica sobre la composición y diversidad de macroinvertebrados acuáticos en el río Cofre (sistema lótico andino colombiano). Revista Biodiversidad Neotropical. 4 (2): 113-123.

Guzmán-Colis, G., Tthalasso, F., Ramírez-López, E., RodríguezNarciso, S., Guerrero-Barrera, A y Avelar-González, F. 2011. Evaluación espaciotemporal de la calidad del agua del río San Pedro en el estado de Aguascalientes, México. Revista Internacional de Contaminación Ambiental. 27(2): 89-102.

Henne L.J., Schneider D.W. y Martínez-Rivera L.M. 2002. Rapid assessment of organic pollution in a west-central Mexican river using a family-level biotic index. Journal of Environmental Planning and Management. 45 (5), 613-632.

Hernández-Vargas, O. 2017. Calidad del agua de dos cuencas tributarias del RíoTuxcacuesco, Jalisco. (Tesis de Licenciatura). Universidad de Guadalajara. Centro Universitario de la Costa Sur. Autlán de Navarro, Jalisco, México. 159 pp.

Mancilla-Villa, O. R., Fregoso-Zamorano, B. E., Hueso-Guerrero, E. J., Guevara-Gutiérrez, R. D., Palomera-García, C y OlguínLópez, J. L. 2017. Concentración iónica y metales pesados en el agua de riego de la cuenca de río Ayuquila-TuxcacuescoArmería. IDESIA. 35(3): 115-123.

Martínez, M., y Fernández, R., D. 2010. Hidrología aplicada a las pequeñas obras hidráulicas. SAGARPA. Colegio de Posgraduados. $20 \mathrm{pp}$.

Merrit R.W. y Cummins K.W. 1996. An introduction of the aquatic insects of North America. 3a Ed. Dubuque: Kendall/Hunt. Michigan, EUA, 862 pp.

Meza, S., Sepúlveda, S. 2012. Efecto de la conductividad eléctrica y nivel del agua sobre la reproducción de la trucha xenocara (Ancistrus triradiatus, Eigenmann 1918) bajo condiciones experimentales. Veterinária e Zootecnia. 6(1): 37-46.

Meza-Rodríguez, D., Martínez-Rivera, L. M., Mercado-Silva, N., García de Jalón-Lastra, D., González del Tánago-Del Río, M., Marchamelo-Sacristán, M. y De la Mora-Orozco, C. 2017. Régimen natural de caudales del río Ayuquila-Armería en el occidente de México. Terra Latinoamericana. 35: 203-217.

Mora-Bueno, D., Sánchez-Peña, L., Del Razo, L. M., GonzálezArias, C., Medina-Díaz, I., Robledo-Marenco, M y RojasGarcía, A. 2012. Presencia de arsénico y coliformes en agua potable del municipio de Tecuala, Nayarit, México. Revista Internacional de Contaminación Ambiental. 28(2): 127-135.

Moya, N., Domínguez, E., Goitia, E. y Oberdoff, T. 2011. Desarrollo de un índice multimétrico basado en macroinvertebrados acuáticos para evaluar la integridad biológica en ríos de los valles interandinos de Bolivia. Asociación Argentina de Ecología; Ecología Austral. 21(2): 7-20.

Muñoz-Nava, H., Suárez-Sánchez, J., Vera-Reyes, A., Baumann, J., Carreón-Coca, M. y Montealegre-Muñoz, R. 2013. Relación entre el nitrógeno y las microcuencas del río Zahuapan, México. Tecnología y ciencias del agua. 4(4): 47-61.

Neal, C., House, W. A., Jarvie, P. H., Neal. M., Hill, L. y Wickham, H. 2006. The water quality of the river Dunn and the Kennet and Avon canal. Journal of Hydrology. 330: 155-170.

NORMA MEXICANA. 1987. NMX-AA-42-1987, Calidad del agua-determinación del número más probable (NMP) de coliformes totales, coliformes fecales (termotolerantes) y Escherichia coli presuntiva. Secretaría de Desarrollo Urbano y Ecología. Diario Oficial de la Federación, México.

Olguín, E., González-Portela, R., Sánchez, G., Zamora, G y Owen, T. 2010. Contaminación de ríos urbanos: El caso de la subcuenca del río Sordo en Xalapa, Veracruz, México. Revista Latinoamericana de Biotecnología Ambiental y Algal. 1(2):178-190.

Olivas-Enríquez, E., Flores-Margez, J. P., Serrano-Alamillo, M., Soto-Mejía, E., Iglesias-Mejía, J., Salazar-Sosa, E. y FortisHernández, M. 2011. Indicadores fecales y patógenos en agua descargada al Río Bravo. Terra Latinoamericana. 29(4): 449-457.

Ortiz-Vega, M. I., Can-Chulim, Á., Romero-Bañuelos, C. A., CruzCrespo, E y Madueño-Molina, A. 2019. Calidad del agua para uso agrícola del río Mololoa, México. Terra Latinoamericana. 37: 185-195

Palomera-García, C. 2012. Effects of land-use activities in the Ayuquila River's fauna (Tesis doctoral). Simon Fraser University, Canada. 179 pp.

Pucci, G., Acuña, Jy Pucci, H. 2013. Contaminación microbiológica por enterobacterias y coliformes totales de la playa de Stela Maris, Comodoro Rivadavia, Argentina, derivada de los efluentes cloacales. Higiene y Sanidad Ambiental. 13 (5): 1102-1107.

Ramírez, E., Robles, E., Sainz, G y Ayala, R. 2009. Calidad microbiológica del acuífero de Zacatepec, Morelos, México. Revista Internacional de Contaminación Ambiental. 25(4): 247-255.

Redondo, M. y Arias, M. 2011. Comparación de métodos para el análisis de coliformes totales y fecales en muestras de agua mediante la técnica de Número Más Probable (NMP). UNED Research Journal. 3(2): 41-43.

Robles, E., Ramírez, E., Durán, A., Martínez, M y González, M. 2013. Calidad bacteriológica y fisicoquímica del agua del acuífero Tepalcingoaxochiapan, Morelos, México. Avances en Ciencias e Ingeniería. 4 (1): 19-28.

Roldan-Pérez, G. 2016. Los macroinvertebrados como bioindicadores de la calidad del agua: cuatro décadas de desarrollo en Colombia y Latinoamérica. Revista de la Academia Colombiana de Ciencias Exactas, Físicas y Naturales. 40(155): 254-274.

Santana E., Navarro S., Martínez L.M., Aguirre A., Figueroa P. y Aguilar C. 1993. Contaminación, aprovechamiento y conservación de los recursos acuáticos del río Ayuquila, Reserva de la Biosfera Sierra de Manantlán, Jalisco-Colima. Tiempos de Ciencia. 30: 29-38. 
Sánchez, D., y Cañón, J. 2010. Análisis documental del efecto de vertimientos domésticos y mineros en la calidad del agua del río Condoto (Chocó, Colombia). Gestión y Ambiente. 13(3): 115-130.

Sardiñas, O., Chiroles, S., Fernández, M., Hernández, Y y Pérez, A. 2006. Evaluación fisicoquímica y microbiológica del agua de la presa El Cacao (Cotorro, Cuba). Higiene y Sanidad Ambiental. 6: 202-206.

SEMARNAT.1996. Norma Oficial Mexicana NOM-001-ECOL-1996. Establece los límites máximos permisibles de contaminantes en las descargas de aguas residuales, en aguas y bienes nacionales. Secretaría de Medio Ambiente, Recursos Naturales y Pesca. Diario Oficial de la Federación. 23 de abril de 1995. Ciudad de México, México, 35 pp.

Sharpley, A. 2010. Manejo de fósforo en sistemas de producción agrícola ambientalmente sostenible: desafíos y oportunidades. Informaciones Agronómicas. 15-23.
Sierra C.A. 2011.Calidad del agua, evaluación y diagnóstico. Universidad de Medellín. Medellín, Colombia, 456 pp.

Solís-Garza, G., Villalba-Atondo, A. I., Nubes-Ortiz, G., Del CastilloAlarcón, J. M. y Meraz-Acosta, F. A. 2011. Fisicoquímica del agua superficial y sedimento en el río Santa Cruz, Sonora, México. Biotecnia. 13 (1), 3-9.

Terneus, E., Hernández, K y Racines, M. 2012. Evaluación ecológica del río Lliquino a través de macroinvertebrados acuáticos, Pastaza-Ecuador. Revista de Ciencias. 16: 31-45.

Viloria, H.; Méndez-Natera, J.R. 2011. Relación entre la conductividad eléctrica, $\mathrm{pH}$ del agua de remojo, germinación de semillas y crecimiento de plántulas de maíz (Zea mayz L.) bajo dos condiciones experimentales. Scientia Agropecuaria. 2(4): 213-228.

Weigel B.M., Henne L.J., Martínez-Rivera L.M. 2002. Macroinvertebrate-based index of biotic integrity for protection of streams in west-central Mexico. Journal of North American Benthological Society. 21(1), 686-700. 